Ertugrul Dalkic • Cem Kuscu • Ceren Sucularli • Iraz T. Aydin · Kamil C. Akcali · Ozlen Konu

\title{
Alternatively spliced Robo2 isoforms in zebrafish and rat
}

Received: 18 October 2005 / Accepted: 13 March 2006 / Published online: 20 April 2006

C) Springer-Verlag 2006

\begin{abstract}
Robo2, a member of the robo gene family, functions as a repulsive axon guidance receptor as well as a regulator of cell migration and tissue morphogenesis in different taxa. In this study, a novel isoform of the zebrafish robo2 (robo2 tv2), which included an otherwise alternatively splice $\bar{d}$ exon (CAE), has been characterized. Robo2 tv2 is expressed differentially in most non-neuronal tissues of adult zebrafish whereas robo2_tvl expression to a great extent is restricted to the brain and eye. In zebrafish, robo2_tv2 exhibits a very-low-level basal expression starting from 1 day post fertilization until the mid-larval stages, at which time its expression increases dramatically and could be detected throughout adulthood. Our findings demonstrate that the amino acid sequence coded by CAE of the robo2 gene is highly conserved between zebrafish and mammals, and also contains conserved motifs shared with robo1 and robo4 but not with robo3. Furthermore, we provide an account of differential transcription of the CAE homolog in various tissues of the adult rat. These results suggest that the alternatively spliced robo2 isoforms may exhibit tissue specificity.
\end{abstract}

Keywords Danio rerio $\cdot$ Rattus norvegicus $\cdot$ robo2 . Alternative splicing $\cdot$ Differential expression

Communicated by M. Hammerschmidt

Electronic Supplementary Material Supplementary material is available for this article at http://dx.doi.org/10.1007/s00427006-0070-y

E. Dalkic $\cdot$ C. Kuscu $\cdot$ C. Sucularli · I. T. Aydin

K. C. Akcali · O. Konu $(\bowtie)$

Department of Molecular Biology and Genetics,

Bilkent University,

06800 Ankara, Turkey

e-mail: konu@fen.bilkent.edu.tr

Tel.: +90-312-2902123

Fax: +90-312-2665097

\section{Introduction}

Robo family genes, which belong to the immunoglobulin superfamily of receptors, play evolutionarily conserved roles in invertebrates and vertebrates with respect to axon guidance and cell migration (Seeger et al. 1993; Yuan et al. 1999; Marillat et al. 2002; Bagri et al. 2002; Long et al. 2004; Sundaresan et al. 2004). Within this family, the vertebrate robo1, 2, and 3 are known to guide neuronal cell migration (Wong et al. 2002; Sundaresan et al. 2004) while robo4 is specifically involved in angiogenesis during embryonic development (Park et al. 2003).

A common characteristic of immunoglobulin superfamily receptors is the frequent alternative splicing events they undergo (Brummendorf and Lemmon 2001). For example, alternative splicing of robol in the mouse has been shown to generate two different isoforms, robol and dutt1, which show differential expression patterns. The dutt 1 isoform is widely expressed in development and is also present in adult tissues whereas the robol isoform is expressed strictly in embryonic brain, eye, and kidney tissues (Clark et al. 2002). In addition, rig1 (robo3) has different isoforms, which are structurally and functionally distinct (Jen et al. 2004; Camurri et al. 2005). However, no experimentally confirmed alternative splicing event has yet been reported in the literature for robo 2 or robo 4.

The zebrafish, a well-established model organism in the developmental genetics field, provides a multitude of opportunities to analyze the extent of alternative splicing events in a stage- and tissue-specific manner (Kimmel et al. 1995; Shen et al. 2002). Zebrafish express all four robo paralogs starting from embryogenesis (Lee et al. 2001; Challa et al. 2001; Bedell et al. 2005). Among these, the zebrafish robo3 gene has been recently shown to produce two distinct variants that function in motor axon pathfinding and dorsoventral cell fate specification, respectively (Challa et al. 2005). Potential alternative splicing events in other zebrafish robo members remain unexplored.

In zebrafish, robo2/astray is responsible for the proper retinal axon guidance, anterior-posterior pathfinding, midline crossing, and fascicular retinal projection (Fricke 
et al. 2001). The predicted amino acid sequence based on the full-length robo 2 cDNA sequence (NM_131633; Lee et al. 2001) indicates that the robo 2 protein has an extracellular domain comprising five immunoglobulin domains and three fibronectin type III domains, a single transmembrane domain, and a cytoplasmic domain containing several conserved motifs. The neuronal expression of robo 2 mRNA in zebrafish is present at $12 \mathrm{~h}$ postfertilization (hpf) and continues through $72 \mathrm{hpf}$ while the expression of other robo paralogs, robol and robo3, are diminished by that time (Lee et al. 2001). In addition to neuronal expression, zebrafish robo family mRNAs are present in non-neuronal tissues including pectoral fin buds during the first 3 days of development (Lee et al. 2001). However, the larval, juvenile, and adult expression pattern or function of robo 2 in non-neuronal cells of zebrafish is not well known. On the other hand, several studies have reported the expression pattern and functional contribution of robo2 in non-neuronal tissues in chicken, rodents, and humans (Piper et al. 2000; Nagase et al. 2000; Vargesson et al. 2001; Anselmo et al. 2003; Greenberg et al. 2004; Grieshammer et al. 2004). A comprehensive study focusing on the human homolog of robo2 (KIAA1568) expression has indicated that robo2 is expressed in various fetal and adult tissues but is excluded from adult pancreas, heart, liver, and skeletal muscle (Nagase et al. 2000). These findings suggest that robo 2 expression may be temporally and spatially compartmentalized.

Our primary aim was to test the presence of alternatively spliced forms of robo2 and, if any such forms exist, to characterize their developmental and tissue-specific expression pattern. We also investigated whether these splicing events were conserved between fish and mammals using the rat as a model system. Our findings indicated the presence of a highly conserved alternative exon (herein named CAE) located in the cytoplasmic portion of the robo2 mRNA. Reverse transcriptase-polymerase chain reaction (RT-PCR) results showed that CAE was more likely to be spliced out in neuronal than in non-neuronal tissues of both organisms. Furthermore, CAE was highly conserved across the paralogs robo1, robo2, and robo 4 but was not incorporated into the robo3 gene structure. These findings implicated CAE in the production of multiple robo 2 proteins which may perform different functions in neuronal and non-neuronal tissues.

\section{Materials and methods}

Fish husbandry

Zebrafish (Danio rerio), purchased from pet-shops or provided by the University of Oregon at Eugene, OR, USA (AB strain), were kept and raised using standard methods. The fish were maintained on a cycle of a 14-h day and a 10-h night at $28.5^{\circ} \mathrm{C}$. Before they were killed, the animals were anesthetized with an overdose of tricaine solution
$(0.12 \%)$. Adult fish were dissected using sterile equipment to obtain different tissues. The embryos were collected in the morning following the setup and maintained in plates filled with system water. Embryos, larvae, and juvenile fish were staged according to hours post-fertilization (hpf), days post-fertilization (dpf), and relevant morphological criteria (Kimmel et al. 1995).

\section{Animals}

Nine-week-old, 200 to 250 -g male or female SpragueDawley rats were used. They were housed under controlled environmental condition $\left(22^{\circ} \mathrm{C}\right)$ in the animal holding facility of Bilkent University, Turkey on a 12-h light and 12-h dark cycle. All of the animals received care according to the criteria outlined in the "Guide for Care and Use of Laboratory Animals" prepared by the National Academy of Science and this study protocol complied with Bilkent University's guidelines on humane care and use of laboratory animals.

Comparison of zebrafish robo2 sequences in NCBI and Ensembl databases

A complete robo2 genomic sequence is not currently available. However, the Ensembl database contains a predicted transcript (Ensdart00000014877; http://www. ensembl.org; WTSI Zv5; Scaffold791) which partially corresponds to zebrafish robo2 (NM_131633). Several NCBI zebrafish ESTs, which are aligned primarily to the $5^{\prime}$ and $3^{\prime}$ ends of the robo2 cDNA sequence (NM_131633), could also be detected (CK686381.1, AL920855.1, CN173823.1, CN318984.1, and AI437295.1). The exon/ intron structure of Ensdart00000014877 in the genomic assembly was analyzed in further detail by comparing it with the corresponding Genscan prediction. The most 3' end of Genscan00000032249 (corresponding to the zebrafish chromosome 15: 40,471,855 to 40,472,040 bp; www. ensembl.org, v37) exhibited a potential alternative exon which was further pursued in the present study ( $\mathrm{S} 1 \mathrm{a}$ in "Appendix").

Rat robo2 sequences in NCBI and Ensembl databases

A full length rat robo2 mRNA sequence was computationally predicted by NCBI (XM_213677). In the Ensembl database, several computationally predicted isoforms have been reported (S1b in "Appendix"); these differed in their length and their usage of alternative exons including the rat homolog of CAE (Ensrnot00000044621, Ensrnot0000 0043725, Ensrnot00000042437, Ensrnot00000050397, Ensrnot00000047577, Ensrnot00000046648, Q9QZI3 RAT; Chromosome 11). 
Orthologous CAE in genomic assembly databases

The human, rat, chimpanzee, dog, and chicken homologs of the zebrafish robo2 protein sequences were extracted from public databases (GenBank, http://www.ncbi.nlm.nih. gov; Ensembl, http://www.ensembl.org). Orthologs of CAE mapped, in general, to Genscan predicted putative exonic sites near the $3^{\prime}$ end of robo2 [human: Chr. 3: 77,764,315:77,764,497 bp, (Genscan00000045332); chimpanzee: Chr. 3: 79,987,335:79,987,517 bp, (Genscan000 00073054); dog: Chr. 31: 12416150: 12416332 bp, (Genscan00000000765)] for all species examined, except chicken and rat. For chicken, Ensembl provided Ensgalt00000025020, which included the $3^{\prime}$ end of robo2. Although this transcript did not contain CAE, its corresponding Genscan sequence [Chr. 1: 90,811,644: 90,811,826 bp, (Genscan00000007903)] incorporated a sequence highly orthologous to zebrafish $\mathrm{CAE}$. In rats, robo2 CAE was already annotated as transcribed based on computational prediction algorithms, although this was not shown experimentally (S1b in "Appendix"). There has been no EST evidence for CAE for any of the aforementioned organisms including the rat.
Primer design

To detect whether the conserved alternative exon (CAE) was expressed in a developmental and/or tissue-specific manner in zebrafish, primers have been designed against various exons in Ensdart00000014877, NM 131633, and the genomic region corresponding to $\mathrm{CAE}$ (Chr. 15: 40,471,855:40,472,040; S1a in "Appendix"; Table 1; Fig. 3). In addition, rat-specific primers were designed to amplify the rat homolog of zebrafish $\mathrm{CAE}$ and the flanking sequences in the neighboring exons ( $\mathrm{S} 1 \mathrm{~b}$ in "Appendix"; Table 1; Fig. 6). Degenerate primers targeting the conserved regions of the $\mathrm{CAE}$ and the flanking sequences (MAMF-MAMRO primer pair) also were used particularly for sequencing purposes (Table 1).

\section{Total RNA extraction}

Dissected fish and rat tissues and embryos were kept in the RNAlater solution (Qiagen, USA, Cat \# 7020) or in liquid nitrogen before performing RNA isolations. All material and solutions were treated with diethyl pyrocarbonate to prevent RNase contamination. RNA extraction was performed from zebrafish embryos of various dpfs in an ascending order from embryonic to juvenile stages. RNA

Table 1 Robo2, mipep, and cyclophilin primer sequences and conditions used for RT-PCR for zebrafish and rat cDNAs amplification and sequencing

\begin{tabular}{|c|c|c|c|c|c|c|}
\hline Name & Sequence $\left(5^{\prime}-3^{\prime}\right)$ & & & & & \\
\hline NCBI1F & CTCGGCAGCCTGCAGATCAA & & & & & \\
\hline $\mathrm{NCBI} 2 \mathrm{~F}$ & AAGACTTCCGCGATGTGATG & & & & & \\
\hline $\mathrm{E} 21 \mathrm{~F}$ & CCTTAGTGTCTGACATCGAG & & & & & \\
\hline E22R & GGACGAGGTCTATGAGGAA & & & & & \\
\hline AER & ATCATCTTCTGAAGCCGAGC & & & & & \\
\hline RATF & CCCCTCAGAGCACTAGACCA & & & & & \\
\hline RATRI & TCCATCCAGCCTAAAACCAG & & & & & \\
\hline RATRO & TCCCTCCGATGAGGTAACAC & & & & & \\
\hline MAMF & GAGCACT(G/A)GACCAGAC(T/A)CC & & & & & \\
\hline MAMRO & T(G/T)GGTCCATCCG(C/T)CCTC & & & & & \\
\hline MIPEPF & ACATCCTCAGAGAATTGCAG & & & & & \\
\hline MIPEPR & GGTGCTGGATCTCGATGTT & & & & & \\
\hline CYCF & GGGAAGGTGAAAGAAGGCAT & & & & & \\
\hline CYCR & GAGAGCAGAGATTACAGGGT & & & & & \\
\hline Primer pair & Denaturation & Cycle number & Denaturation & Annealing & Extension & Extension \\
\hline NCBI1F-E22R & $93^{\circ} \mathrm{C}, 2 \mathrm{~min}$ & 40 & $93^{\circ} \mathrm{C}, 10 \mathrm{~s}$ & $56^{\circ} \mathrm{C}, 30 \mathrm{~s}$ & $72^{\circ} \mathrm{C}, 3 \mathrm{~min}$ & $72^{\circ} \mathrm{C}, 10 \mathrm{~min}$ \\
\hline NCBI1F-AER & $93^{\circ} \mathrm{C}, 2 \mathrm{~min}$ & 40 & $93^{\circ} \mathrm{C}, 10 \mathrm{~s}$ & $55^{\circ} \mathrm{C}, 30 \mathrm{~s}$ & $72^{\circ} \mathrm{C}, 4 \mathrm{~min}$ & $72^{\circ} \mathrm{C}, 10 \mathrm{~min}$ \\
\hline NCBI2F-E22R & $93^{\circ} \mathrm{C}, 2 \mathrm{~min}$ & 40 & $93^{\circ} \mathrm{C}, 10 \mathrm{~s}$ & $56^{\circ} \mathrm{C}, 30 \mathrm{~s}$ & $72^{\circ} \mathrm{C}, 3 \mathrm{~min}$ & $72^{\circ} \mathrm{C}, 10 \mathrm{~min}$ \\
\hline NCBI2F-AER & $93^{\circ} \mathrm{C}, 2 \mathrm{~min}$ & 40 & $93^{\circ} \mathrm{C}, 10 \mathrm{~s}$ & $55^{\circ} \mathrm{C}, 30 \mathrm{~s}$ & $72^{\circ} \mathrm{C}, 4 \mathrm{~min}$ & $72^{\circ} \mathrm{C}, 10 \mathrm{~min}$ \\
\hline E21F-E22R & $95^{\circ} \mathrm{C}, 5 \mathrm{~min}$ & 30 & $95^{\circ} \mathrm{C}, 30 \mathrm{~s}$ & $58^{\circ} \mathrm{C}, 30 \mathrm{~s}$ & $72^{\circ} \mathrm{C}, 30 \mathrm{~s}$ & $72^{\circ} \mathrm{C}, 10 \mathrm{~min}$ \\
\hline E21F-AER & $95^{\circ} \mathrm{C}, 5 \mathrm{~min}$ & 30 & $95^{\circ} \mathrm{C}, 30 \mathrm{~s}$ & $58^{\circ} \mathrm{C}, 30 \mathrm{~s}$ & $72^{\circ} \mathrm{C}, 30 \mathrm{~s}$ & $72^{\circ} \mathrm{C}, 10 \mathrm{~min}$ \\
\hline RATF-RATRI & $95^{\circ} \mathrm{C}, 5 \mathrm{~min}$ & 30 & $94^{\circ} \mathrm{C}, 30 \mathrm{~s}$ & $55^{\circ} \mathrm{C}, 30 \mathrm{~s}$ & $72^{\circ} \mathrm{C}, 30 \mathrm{~s}$ & $72^{\circ} \mathrm{C}, 10 \mathrm{~min}$ \\
\hline RATRF-RATRO & $95^{\circ} \mathrm{C}, 5 \mathrm{~min}$ & 30 & $94^{\circ} \mathrm{C}, 30 \mathrm{~s}$ & $58^{\circ} \mathrm{C}, 30 \mathrm{~s}$ & $72^{\circ} \mathrm{C}, 30 \mathrm{~s}$ & $72^{\circ} \mathrm{C}, 10 \mathrm{~min}$ \\
\hline MAMF-MAMRO & $95^{\circ} \mathrm{C}, 5 \mathrm{~min}$ & 35 & $95^{\circ} \mathrm{C}, 30 \mathrm{~s}$ & $55^{\circ} \mathrm{C}, 30 \mathrm{~s}$ & $72^{\circ} \mathrm{C}, 30 \mathrm{~s}$ & $72^{\circ} \mathrm{C}, 10 \mathrm{~min}$ \\
\hline MIPEPF-MIPEPR & $95^{\circ} \mathrm{C}, 5 \mathrm{~min}$ & $30 / 35$ & $95^{\circ} \mathrm{C}, 30 \mathrm{~s}$ & $58^{\circ} \mathrm{C}, 30 \mathrm{~s}$ & $72^{\circ} \mathrm{C}, 30 \mathrm{~s}$ & $72^{\circ} \mathrm{C}, 10 \mathrm{~min}$ \\
\hline CYCF-CYCR & $95^{\circ} \mathrm{C}, 5 \mathrm{~min}$ & 25 & $95^{\circ} \mathrm{C}, 30 \mathrm{~s}$ & $58^{\circ} \mathrm{C}, 30 \mathrm{~s}$ & $72^{\circ} \mathrm{C}, 30 \mathrm{~s}$ & $72^{\circ} \mathrm{C}, 10 \mathrm{~min}$ \\
\hline
\end{tabular}


was extracted based on the instructions of the manufacturer (Qiagen Rneasy Mini Kit, USA, Cat\# 74124) from five embryos, one to five larvae, or one juvenile fish per dpf, and from multiple adult tissues of two or more zebrafish (i.e., eye, brain, liver, muscle, heart, caudal fin, pectoral fin, dorsal fin, spleen, gills, digestive tract, and ovaries). Total RNA from different rat tissues (brain, liver, kidney, lung, spleen, heart, skeletal muscle, testes, and ovary) was isolated by using TriPure Isolation Reagent (Roche Diagnostics, USA, Cat \# 11667157001) according to the instructions of the manufacturer.

RT-PCR analysis and sequencing of robo2 products

Equal amounts of total RNA from zebrafish embryo, larvae, juveniles, and various tissues of adult fish and rat were converted into the first strand cDNA according to the manufacturer's protocols (Fermentas, Canada). PCR reactions were performed using the appropriate primer pairs for each cDNA sample (Techne, ftGENE2D, Cambridge, England). Each reaction contained a total of $25-\mu$ reaction volume that included $1 \mu \mathrm{g}$ cDNA, 10 pmol reverse and forward primers, $0.5 \mathrm{ml} \mathrm{dNTP}(0.2 \mathrm{mM}$, each $), 1.5 \mathrm{mM}$ $\mathrm{MgCl}_{2}, 2.5 \mu \mathrm{l} 10 \times$ PCR buffer, and 1 unit Taq DNA polymerase (Fermentas, Canada). PCR conditions for each primer pair are indicated in Table 1. Size-matched amplicons with or without CAE from zebrafish and rat (for primer pairs, see Table 1) were sequenced (Iontek Sequencing Facility, Istanbul, Turkey; ABI PRISM 310 Genetic Analyzer, Applied Biosystems, USA) upon gel extraction and purification (QIAquick Gel Extraction Kit, Cat \# 28704).

\section{Results and discussion}

Identification and conservation of the putative alternative exon

Transcript and genomic information from the Ensembl database (Ensdart00000014877 and ENSDARG00000014 891 in Chr.15: 40,369,682-40,475,421; WTSI Zv5) were used to identify possible alternatively spliced exons not present in the current robo 2 cDNA sequence but predicted by Genscan (see "Materials and methods" for details). We previously had observed that primers targeted to a region spanning 3,955-4,126 bp (E21F-E22R; Table 1) of robo2 mRNA sequence (NM_131633) resulted in amplification of two different products in zebrafish, suggestive of an alternative splicing event in robo2. A 186-bp sequence, herein called CAE that corresponds to a region in chromosome 15 (from 40,471,855 to $40,472,040 \mathrm{bp}$ and in between 21st and 22nd exons of Ensdart00000014877), has accordingly been identified. Distant homology searches for the presence of a CAE homolog in the human, chimpanzee, dog, rat, and chicken genomic sequences suggested that CAE was likely to be expressed, although previously no ESTs with CAE had been reported (see "Materials and methods" for details). Unlike in vertebrates, no homologous sequence to CAE could be identified for invertebrates.

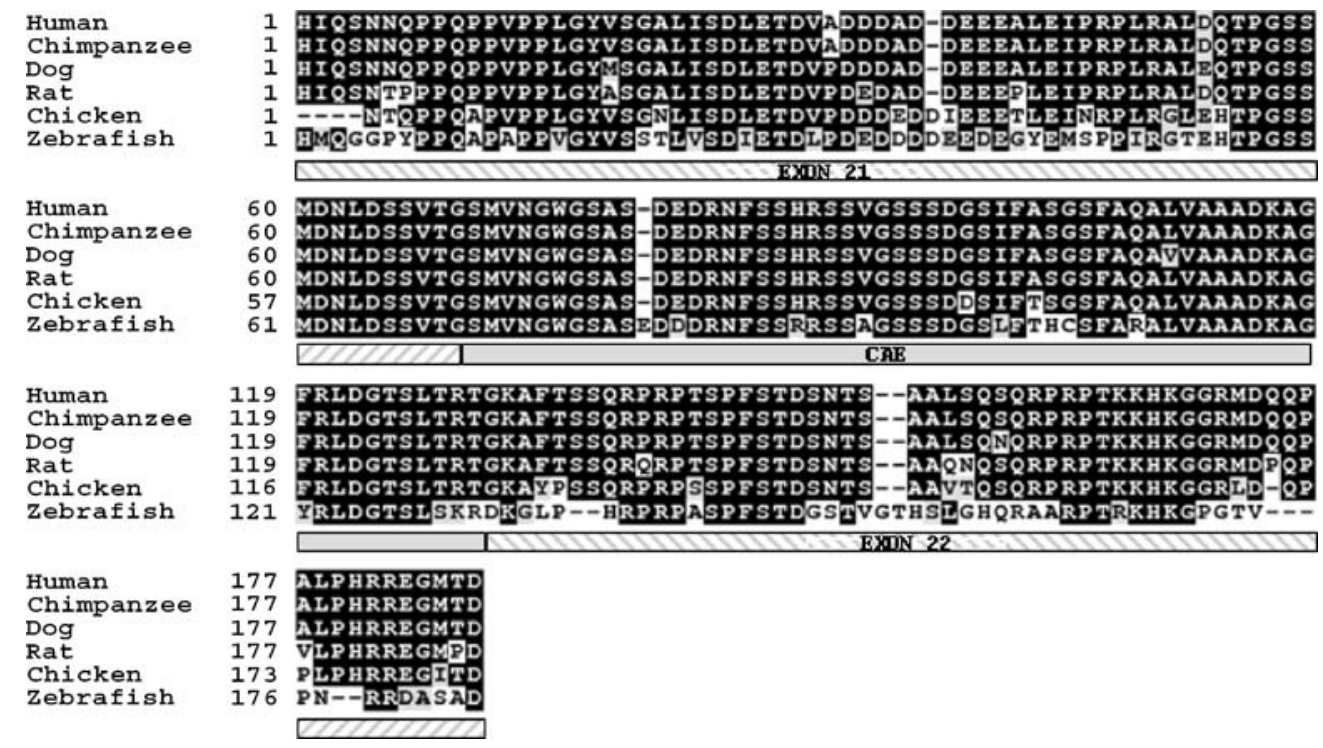

Fig. 1 Multiple alignment of the robo2 protein sequences for the human, rat, chimpanzee, dog, chicken, and zebrafish was performed using ClustalW 1.8 in BCM Search Launcher (http://www. searchlauncher.bcm.tmc.edu/) and then visualized using BOXSHADE 3.1 (http://www.ch.embnet.org/software/BOX form.html). The conserved alternative exon has been manually translated and incorporated into the protein sequence when not included in the original NCBI database sequences. The accession numbers and location of the amino acid used at the first position of the alignment are given for each species in parentheses: human robo2 (NP 002933; 1,186), rat robo2 (XP 213677; 1,313), chimpanzee robo-2 (XP 516591; 1,121), dog robo2 (XP 544815; 1,186),

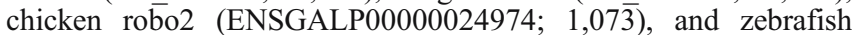
robo2 (NP_571708; 1,242). The location of CAE and those of the exons 21 and 22 are depicted using shaded boxes 

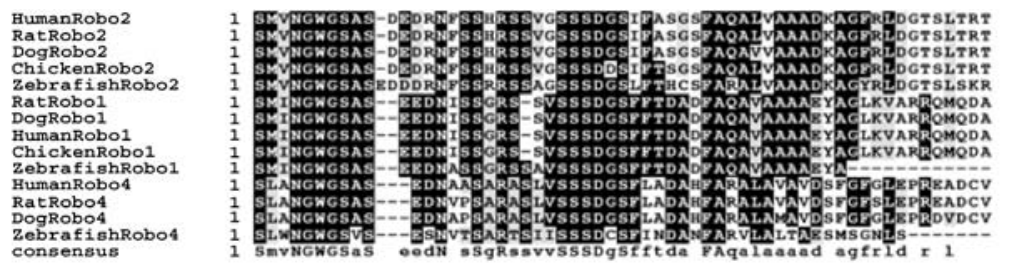

b

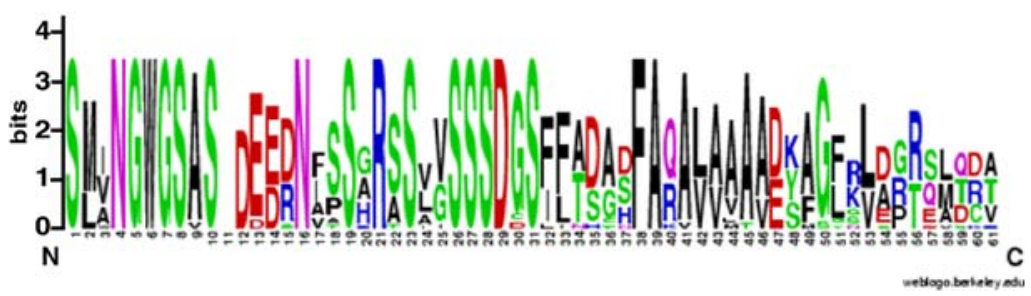

Fig. 2 a Multiple alignment of the protein sequences of robo proteins from different species. Human: robol (NP 002932, residues 1369-1426), robo4 (NP 061928, residues 872-929); rat: robo1 (NP 071524; 1,369-1,426) , robo2 (XP 213677; 1,3821,441), robo4 (NP 852040; 890-947); dog: robo1 (XP 544814.2; 1,420-1,477), robo4 (XP 546424.2, 912-969); zebrafish: robo1 (NP 571556; 1,388-1,434), robo4 (AAQ10890; 1,014-1,064); and chicken: robol (XP_416673; 1,690-1,747). The first position shown

Multiple alignment of robo2 orthologous sequences indicated that the protein sequence of zebrafish CAE was highly similar to that of human (80\%), chimpanzee $(80 \%)$, rat (80\%), dog (78\%), and chicken (80\%) CAE (Fig. 1), whereas the residues from $5^{\prime}$ and $3^{\prime}$ neighboring exons were not as highly conserved ( 5 ' exon: $62 \%$ to human, $62 \%$ to chimpanzee, $62 \%$ to rat, $63 \%$ to dog, and $68 \%$ to chicken; ' 3 ' exon: $43 \%$ to human, $43 \%$ to chimpanzee, $33 \%$ to rat, $43 \%$ to dog, and $41 \%$ to chicken). CAE is predicted to yield an in-frame insertion without stop codons (Fig. 1). It corresponds to a region between the second and the third cytoplasmic domains (between the 1,311th and 1,312th residues of NM 131633) of the zebrafish robo2 protein sequence.

The high sequence homology and preserved reading frame between the orthologous sequences of CAE suggest that this sequence is functionally important and conserved. It also is possible that CAE could be incorporated in multiple robo2 isoforms other than the ones specified in this study. Robo2 as a guidance molecule might be crucial for the regulation of cell-cell and cell-extracellular matrix interactions in adult life.

CAE also was conserved between paralogs robol and robo4, among different vertebrate taxa (Fig. 2a). Robo1, robo2, and robo4 proteins all possessed a highly conserved motif (Fig. 2b) exhibiting greater sequence similarity within the members of a particular paralog (e.g., a paralogspecific submotif: SMVNGWGSAS for robo2, SMINGWGSAS for robo1, and SLABGWGSAS for robo4). On the other hand, the conserved motif shown in Fig. $2 \mathrm{~b}$ was not present in robo3 in any of the organisms.

One evolutionarily plausible hypothesis to explain the differential incorporation of CAE into the functional protein structure of robo family members could involve the exclusion of CAE in robo 3 and tissue-specific inclusion in the alignment corresponds to the initial residue given in the parentheses above. Robo2 CAE sequences are as previously described in Fig. 1. The last row of the multiple alignment refers to a consensus sequence. No protein sequence with high similarity to the consensus motif could be identified for chicken robo 4 in the genome; thus, the chicken robo4 sequence is not aligned. b CAE motif highly conserved among robo paralogs

of CAE in robo2. On the other hand, robo1 and robo4 could contain CAE either constitutively or alternatively, although no EST without CAE has been reported yet. It is possible that the incorporation of the CAE into robol and robo4 protein structure might be crucial for their function, whereas its exclusion could be dispensible or only condition-specifically advantageous, thus leading to functional divergence in other robo paralogs. The alternative usage of CAE in robo paralogs accordingly warrants future functional studies.

a

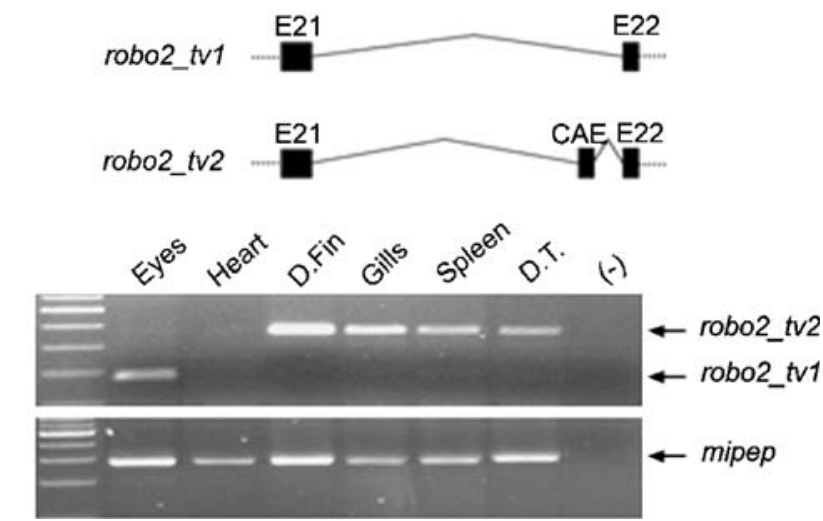

Fig. 3 Genomic representation and differential expression of robo2 alternative isoforms in zebrafish adult tissues. a Genomic representation of robo2 isoforms with or without the CAE. b RT-PCR results with primers E21F-E22R (Table 1) on various tissues of zebrafish adults are shown. The expected size of the amplicon is $172 \mathrm{bp}$ when the alternative exon, CAE, is spliced out while amplicon size increases to $358 \mathrm{bp}$ when CAE is included. The top band of the $100 \mathrm{bp}$ DNA Ladder Plus corresponds to $600 \mathrm{bp}$. c The RT-PCR of the same tissues also was performed for amplification of mipep as a house-keeping gene (expected size, 318 bp) using MIPEP F-R primers 


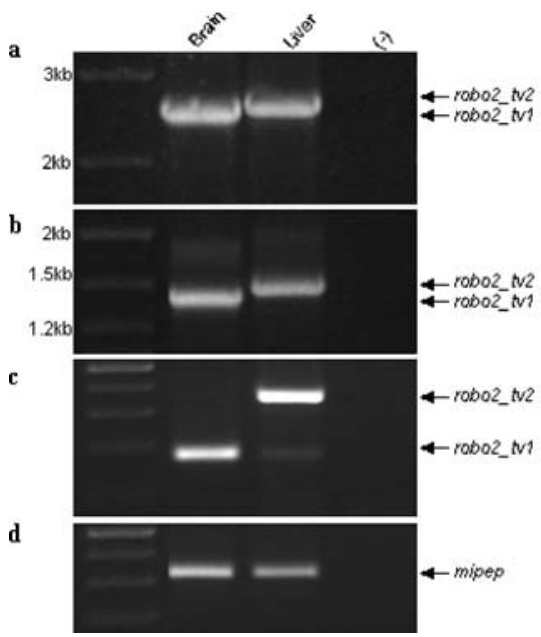

Fig. 4 The expression of robo 2 tv 1 and robo 2 tv2 isoforms on zebrafish brain and liver tissues. a Amplification with forward primer NCBI1F (NM 131633; 1,593-1,613) and the reverse primer E22R. Expected sizes of robo2 tv1 and robo2 tv2 are 2,534 and $2,720 \mathrm{bp}$ in brain and liver, respectively. The first lane corresponds to DNA Ladder Plus. b Amplification with NCBI2F (NM 131633; 2,692-2,712) and the reverse primer E22R. Expected sizes of robo2_tv1 and robo2_tv2 are 1,435 and 1,621 bp in brain and liver, respectively. The first lane corresponds to DNA Ladder Plus. c Amplification with the forward primer E21F (NM 131633; 3,9553,974 ) and the reverse primer E22R (NM 131633; 4,108-4,126). Expected sizes of robo $2 t v 1$ and robo $2 t v \overline{2}$ are 172 and $358 \mathrm{bp}$ in brain and liver, respectively. The first lane corresponds to DNA Ladder Plus; maximum size shown is $500 \mathrm{bp}$. $\mathbf{d}$, h RT-PCR of the

Expression pattern of robo2 isoforms in adult zebrafish and embryonic/larval development

The expression of the transcripts of potential robo2 isoforms encompassing CAE was investigated in a series of zebrafish adult tissues including the eye, heart, spleen,

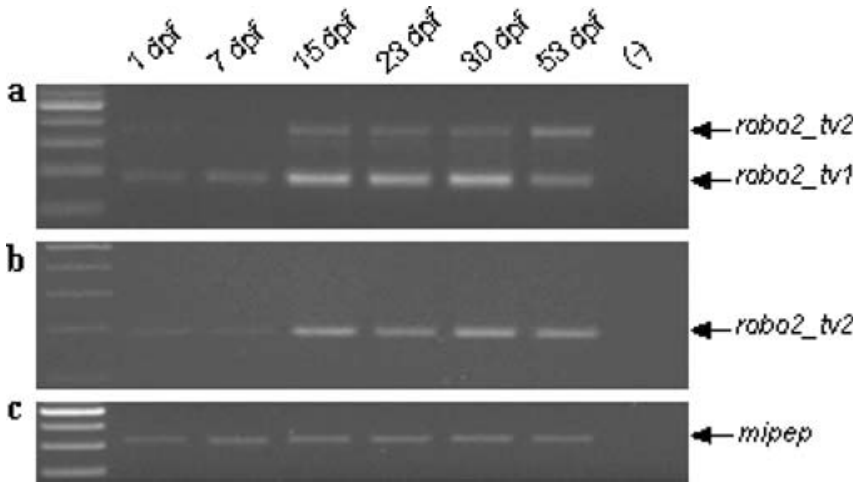

Fig. 5 The expression of robo2 isoforms during zebrafish development. a RT-PCR results with the primer pair E21F-E22R (expected amplicon sizes 172 and 358, with and without CAE, respectively) performed on cDNAs from various stages: $1,7,15,23$, 30 , and $53 \mathrm{dpf}$. The first lane corresponds to 100-bp DNA Ladder Plus (maximum size shown, $600 \mathrm{bp}$ ). b Amplification of RT-PCR results performed on the same set of cDNAs using the primer pair E21F-AER, AER reverse primer specifically targeting a region within CAE. A 100-bp DNA Ladder Plus (maximum size shown, $500 \mathrm{bp}$ ) was used. $\mathbf{c}$ RT-PCR of the same tissues was also performed with MIPEP F-R primers. The first lane corresponds to 100-bp DNA Ladder Plus (maximum size shown, $500 \mathrm{bp}$ )

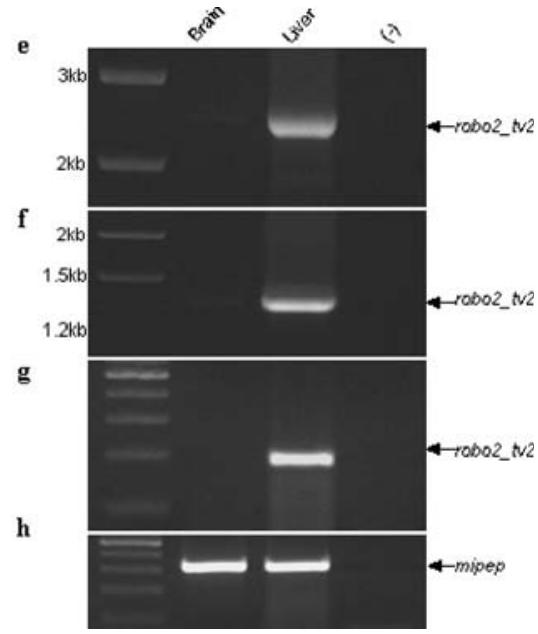

same tissues performed using MIPEP F-R primers (Table 1). The first lane corresponds to $100 \mathrm{bp}$ DNA Ladder; maximum size shown is 500 bp. e Amplification with forward primer NCBI1F (NM 131633; 1,593-1,613) and the reverse primer AER (Table 1). Expected sizes of robo2 tv2 is $2,547 \mathrm{bp}$ in liver. The first lane corresponds to DNA Lad̄er Plus. f Amplification with forward primer NCBI2F (NM 131633; 2,692-2,712) and the reverse primer AER. Expected size of robo2 tv2 is 1,448 bp in liver. The first lane corresponds to DNA Ladder Plus. g Amplification with the forward primer E21F (NM 131633; 3,955-3,974) and the reverse primer AER. Expected size of robo2 tv2 is 185 in liver. The first lane corresponds to DNA Ladder Plus; maximum size shown is $500 \mathrm{bp}$

digestive tract, gills, and dorsal fin together with that of a housekeeping gene mipep (mitochondrial intermediate peptidase) (Fig. 3). Our findings demonstrated that CAE was either skipped or included depending on the tissue type; this produced two alternative forms herein called robo2_tv1 and robo2_tv2, respectively (Fig. 3b). In many of the non-neuronal tissues, robo2 tv2 was expressed at detectable levels while robo 2 tv $\bar{l}$ was predominantly expressed in the eye. Furthermore, the expression of these isoforms was either very low or absent in zebrafish heart (Fig. 3b).

As robo2_tv1 and robo2_tv2 seem to be differentially expressed in neuronal and non-neuronal tissues of zebrafish, brain and liver were used as representative tissues for subsequent experiments. The differential expression pattern of robo2 tv1 and robo2 tv2 in liver and brain was confirmed in RT-PCR reactions performed with three different $5^{\prime}$ forward primers, two of which targeted the extracellular portion of robo2 protein and one targeted the exon 21 preceding the CAE (Table 1; Fig. 4). Our findings indicated that CAE was highly expressed in liver while its expression was absent or negligible in brain (Fig. 4e-g), whereas robo 2 tv 1 was predominant in brain (Fig. 4a-c). Sequencing of both the robo2_tv1- and the robo2_tv2specific PCR products obtained from zebrafish brain and liver tissue cDNAs confirmed the alternative pre-mRNA splicing of CAE in zebrafish (DQ481484, DQ481485). The presence of alternative splicing events concerning exons other than CAE has not been tested in this study; thus, it cannot be excluded. In general, robo2 t $t 22$ expression was 
predominantly present in multiple non-neuronal tissues whereas zebrafish robo2_tv1 isoform could be characterized as neurally enriched although not necessarily neurally restricted.

Considering that the relative amount of robo2 $t v 1$ and robo2 tv2 expression varied among adult tissues, the possibility of differential expression during embryogenesis and at larval-juvenile stages was also assessed (Fig. 5). Our results indicated that robo2_tv2 expression exhibited a dramatic increase starting from the mid-larval stages while it was hardly detectable during embryogenesis or early larval stages (Fig. 5a,b). Robo2 tv1 expression, present at higher levels during embryonic and early larval stages, also displayed an induction later in development (Fig. 5a).

The two isoforms, robo2 $t v 1$ and robo2 $t v 2$, identified in the present study differ in their use of $\overline{\mathrm{CAE}}$, which is located in the intracellular part of the protein. The extracellular Ig domains allow the binding of slit leucinerich regions, whereas the intracellular portion of robo2 relays the signal (Chen et al. 2001; Battye et al. 2001; Nguyen Ba-Charvet et al. 2001; Liu et al. 2004). It is interesting that while the extracellular domain of robo2 is evolutionarily conserved, the intracellular domain has been shown to be divergent (Lee et al. 2001). The high-level expression of the robo 2 tv 2 and the scanty expression of the robo2 tv1 in non-neuronal tissues suggest the possible compartmentalization of the cell migratory and axon guidance roles in the developing animal. Further studies are needed to assess whether robo2 isoforms that contain $\mathrm{CAE}$ could have a role beyond regulating the cytoskeleton; they perhaps act as transcriptional factors (Couch et al. 2004) regulating the extent and timing of the molecular events leading to tissue morphogenesis.

Expression pattern of robo2 isoforms in the adult rat

Rat robo2 mRNA (XM_213677) was reported to include the homolog of the zebrafish CAE, although some of the Ensembl-predicted isoforms differed in their usage of it (Ensrnot00000044621, Ensrnot00000043725, Ensrnot00 000042437, Ensrnot00000050397). To determine whether robo 2 is also differentially and alternatively expressed in rats, RT-PCR from several rat tissues, including brain, skeletal muscle, heart, liver, spleen, kidney, lung, testes, and ovary was performed with rat-specific primers, RATF and RATRO (Fig. 6; Table 1). Our results demonstrated that, although the rat homolog of CAE (Fig. 6a) was transcribed in most of the tissues examined, exon skipping was observed to a greater degree in brain and testes (Fig. 6b). On the other hand, the CAE exclusion and inclusion events were comparable to each other in the ovary (Fig. 6b). In addition, nested PCRs on these PCR products performed with the alternative exon-specific primer pair, RATF and RATRI (Table 1), yielded the expected product (Fig. 6c). These findings support the predictions reported in Ensembl in regards to differential incorporation of the rat CAE in robo2 transcripts (DQ481486, DQ481487).
In rats, the expression of the robo2_tv1 isoform without CAE was found predominantly in the brain similar to that of zebrafish. Several distinguishing features between zebrafish and rats were also obvious with regards to the transcription of CAE. The expression of the CAE seemed to be more ubiquitous in rats and, thus, was found in both the neuronal and non-neuronal tissues unlike in zebrafish. Moreover, zebrafish heart contained very little of either isoforms, if any, whereas both were detectable in rats. The expression levels of robo2 t $v 1$ in brain and testis were relatively high compared to other tissues; this expression profile was in accord with the findings of a recent study on alternative splicing capacities of human tissues (Yeo et al. 2004). It has been reported that the brain and testis have the highest levels of exon-skipping events while the ovary is one of the tissues likely to have multiple isoforms simultaneously.

In summary, an alternative splicing event in a highly conserved predicted exon (CAE) in robo 2 genes of zebrafish and rat was identified and experimentally verified (Figs. 1 and 6). CAE of zebrafish was transcribed in most of the non-neuronal tissues while it was either absent or expressed at a very low level in the brain, eye, and heart. Furthermore, the isoform-specific expression of robo2 appeared to be tightly controlled during development such that zebrafish larvae exhibited an expressional induction of robo2_tv1 and robo2_tv2 at around the mid-larval stages. These findings might implicate robo2 $t v 2$ in a role in tissue morphogenesis, during which cell growth/migration is

a

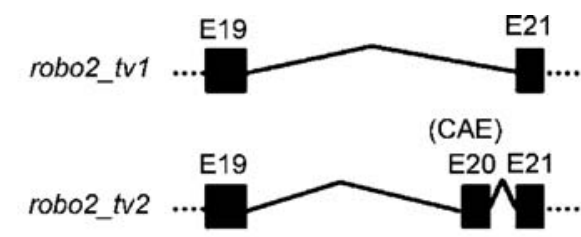

b

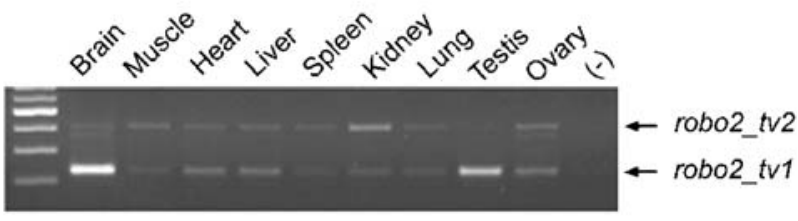

c

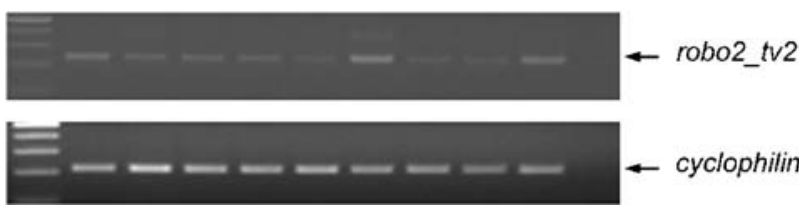

Fig. 6 Genomic representation and differential expression of robo2 alternative isoforms in rat. a Genomic representation of robo2 isoforms b RT-PCR result with primers RATF-RATRO, where RATF targets E19 and RATRO targets E21, on various rat tissues is shown. Expected sizes of the rat robo2 $t v 1$ and robo2 $t v 2$ are $224 \mathrm{bp}$ and $407 \mathrm{bp}$, respectively. The first lane corresponds to 100 bp DNA Ladder Plus (maximum size shown, 600 bp). c Nested PCR result performed with primers RATF-RATRI, where RATRI targets CAE (i.e., E20) in a, using the reactions from RATFRATRO PCR of b (expected band size, 228 bp). A 100-bp DNA Ladder Plus (maximum size shown, $500 \mathrm{bp}$ ) was used. d RT-PCR of the same samples performed with cyclophilin F-R primers. A 100-bp DNA Ladder Plus (maximum size shown, 500 bp) was used 
temporally and spatially regulated. There is unearthed evidence indicating that tissue-type-dependent and, in some cases, neuronal non-neuronal tissue-distinct expression profiles result from alternative splicing events (Fukuda 2003; Shen et al. 2002; Jin et al. 2002; Rahman et al. 2002; Ramming et al. 2000; Hu et al. 1999). Some robo family members have previously been shown to exhibit alternatively spliced isoforms leading to functional divergence within Robo paralogs (Clark et al. 2002; Challa et al. 2005; Camurri et al. 2005). The differential expression of robo2 isoforms in neuronal and non-neuronal tissues could similarly become a source of variation in the function of robo2, possibly directed by different regulatory processes. Our findings strongly point to the existence of multiple robo2 proteins; thus, it is critical to identify which form is involved in a specific developmental process or event in pathogenesis (Latil et al. 2003).

Acknowledgements The authors thank Dr. Kristen Ward and Rasit Ozturk for helpful comments. This research was supported by grants from The Scientific and Technical Research Council of Turkey, TUBITAK (TBAG-2285, 103T03) and Bilkent University to O. Konu. Ertugrul Dalkic has a graduate scholarship from TUBITAK.

\section{References}

Anselmo MA, Dalvin S, Prodhan P, Komatsuzaki K, Aidlen JT, Schnitzer JJ, Wu JY, Kinane TB (2003) Slit and robo: expression patterns in lung development. Gene Expr Patterns 3(1):13-19

Bagri A, Marin O, Plump AS, Mak J, Pleasure SJ, Rubenstein JL, Tessier-Lavigne M (2002) Slit proteins prevent midline crossing and determine the dorsoventral position of major axonal pathways in the mammalian forebrain. Neuron 33(2):233-248

Battye R, Stevens A, Perry RL, Jacobs JR (2001) Repellent signaling by slit requires the leucine-rich repeats. J Neurosci 21:4290-4298

Bedell VM, Yeo SY, Park KW, Chung J, Seth P, Shivalingappa V, Zhao J, Obara T, Sukhatme VP, Drummond IA, Li DY, Ramchandran R (2005) Roundabout4 is essential for angiogenesis in vivo. Proc Natl Acad Sci USA 102:6373-6378

Brummendorf T, Lemmon V (2001) Immunoglobulin superfamily receptors: cis-interactions, intracellular adapters and alternative splicing regulate adhesion. Curr Opin Cell Biol 13(5):611-618

Camurri L, Mambetisaeva E, Davies D, Parnavelas J, Sundaresan V, Andrews W (2005) Evidence for the existence of two Robo3 isoforms with divergent biochemical properties. Mol Cell Neurosci 30(4):485-493

Challa AK, Beattie CE, Seeger MA (2001) Identification and characterization of roundabout orthologs in zebrafish. Mech Dev 101(1-2):249-253

Challa AK, McWhorter ML, Wang C, Seeger MA, Beattie CE (2005) Robo3 isoforms have distinct roles during zebrafish development. Mech Dev 122:1073-1086

Chen JH, Wen L, Dupuis S, Wu JY, Rao Y (2001) The N-terminal leucine rich regions in Slit are sufficient to repel olfactory bulb axons and subventricular zone neurons. J Neurosci 21:15481556

Clark K, Hammond E, Rabbitts P (2002) Temporal and spatial expression of two isoforms of the Dutt1/Robo1 gene in mouse development. FEBS Lett 523:12-16

Couch JA, Chen J, Rieff HI, Uri EM, Condron BG (2004) Robo2 and Robo3 interact with Eagle to regulate serotonergic neuron differentiation. Development 131:997-1006
Fricke C, Lee JS, Geiger-Rudolph S, Bonhoeffer F, Chien CB (2001) Astray, a zebrafish Roundabout homolog required for retinal axon guidance. Science 292:507-510

Fukuda M (2003) Molecular cloning and characterization of human, rat, and mouse synaptotagmin XV. Biochem Biophys Res Commun 306(1):64-71

Greenberg JM, Thompson FY, Brooks SK, Shannon JM, Akeson AL (2004) Slit and robo expression in the developing mouse lung. Dev Dyn 230(2):350-360

Grieshammer U, Le M, Plump AS, Wang F, Tessier-Lavigne M, Martin GR (2004) SLIT2 mediated ROBO2 signaling restricts kidney induction to a single site. Dev Cell 6:709-717

Hu Q, Hearn MG, Jin LW, Bressler SL, Martin GM (1999) Alternatively spliced isoforms of FE65 serve as neuron-specific and non-neuronal markers. J Neurosci Res 58(5):632-640

Jen JC, Chan WM, Bosley TM, Wan J, Carr JR, Rub U, Shattuck D, Salamon G, Kudo LC, Ou J, Lin DD, Salih MA, Kansu T, Al Dhalaan H, Al Zayed Z, MacDonald DB, Stigsby B, Plaitakis A, Dretakis EK, Gottlob I, Pieh C, Traboulsi EI, Wang Q, Wang L, Andrews C, Yamada K, Demer JL, Karim S, Alger JR, Geschwind DH, Deller T, Sicotte NL, Nelson SF, Baloh RW, Engle EC (2004) Mutations in a human ROBO gene disrupt hindbrain axon pathway crossing and morphogenesis. Science 304(5676):1509-1513

Jin M, Tanaka S, Sekino Y, Ren Y, Yamazaki H, Kawai-Hirai R, Kojima N, Shirao T (2002) A novel, brain-specific mouse drebrin: cDNA cloning, chromosomal mapping, genomic structure, expression, and functional characterization. Genomics 79(5):686-692

Kimmel CB, Ballard WW, Kimmel SR, Ullmann B, Schilling TF (1995) Stages of embryonic development of the zebrafish. Dev Dyn 203(3):253-310

Latil A, Chene L, Cochant-Priollet B, Mangin P, Fournier G, Berthon P, Cussenot O (2003) Quantification of expression of netrins, slits and their receptors in human prostate tumors. Int J Cancer 103(3):306-315

Lee JS, Ray R, Chien CB (2001) Cloning and expression of three zebrafish Roundabout homologs suggest roles in axon guidance and cell migration. Dev Dyn 221:216-230

Liu Z, Patel K, Schmidt H, Andrews W, Pini A, Sundaresan V (2004) Extracellular Ig domains 1 and 2 of Robo are important for ligand (Slit) binding. Mol Cell Neurosci 26(2):232-240

Long H, Sabatier C, Ma L, Plump A, Yuan W, Ornitz DM, Tamada A, Murakami F, Goodman CS, Tessier-Lavigne M (2004) Conserved roles for slit and robo proteins in midline commissural axon guidance. Neuron 42(2):213-223

Marillat V, Cases O, Nguyen-Ba-Charvet KT, Tessier-Lavigne M, Sotelo C, Chedotal A (2002) Spatiotemporal expression patterns of slit and robo genes in the rat brain. J Comp Neurol 442(2):130-155

Nagase T, Kikuno R, Nakayama M, Hirosawa M, Ohara O (2000) Prediction of the coding sequences of unidentified human genes. XVIII. The complete sequences of 100 new cDNA clones from brain which code for large proteins in vitro. DNA Res 7:273-281

Nguyen Ba-Charvet KT, Brose K, Ma L, Wang KH, Marillat V, Sotelo C, Tessier-Lavigne M, Chedotal A (2001) Diversity and specificity of actions of Slit2 proteolytic fragments in axon guidance. J Neurosci 21:4281-4289

Park KW, Morrison CM, Sorensen LK, Jones CA, Rao Y, Chien CB, Wu JY, Urness LD, Li DY (2003) Robo4 is a vascular-specific receptor that inhibits endothelial migration. Dev Biol 261:251267

Piper M, Georgas K, Yamada T, Little M (2000) Expression of the vertebrate Slit gene family and their putative receptors, the Robo genes, in the developing murine kidney. Mech Dev 94(12):213-217

Rahman L, Bliskovski V, Reinhold W, Zajac-Kaye M (2002) Alternative splicing of brain-specific PTB defines a tissuespecific isoform pattern that predicts distinct functional roles. Genomics 80(3):245-249 
Ramming M, Kins S, Werner N, Hermann A, Betz H, Kirsch J (2000) Diversity and phylogeny of gephyrin: tissue-specific splice variants, gene structure, and sequence similarities to molybdenum cofactor-synthesizing and cytoskeleton-associated proteins. Proc Natl Acad Sci USA 97(18):10266-10271

Seeger M, Tear G, Ferres-Marco D, Goodman CS (1993) Mutations affecting growth cone guidance in Drosophila: genes necessary for guidance toward or away from the midline. Neuron 10:409426

Shen H, Illges H, Reuter A, Stuermer CAO (2002) Cloning, expression, and alternative splicing of neogenin1 in zebrafish. Mech Dev 118:219-223

Sundaresan V, Mambetisaeva E, Andrews W, Annan A, Knoll B, Tear G, Bannister L (2004) Dynamic expression patterns of Robo (Robo1 and Robo2) in the developing murine central nervous system. J Comp Neurol 468(4):467-481
Vargesson N, Luria V, Messina I, Erskine L, Laufer E (2001) Expression patterns of slit and robo family members during vertebrate limb development. Mech Dev 106(1-2):175-180

Wong K, Park HT, Wu JY, Rao Y (2002) Slit proteins: molecular guidance cues for cells ranging from neurons to leukocytes. Curr Opin Genet Dev 12(5):583-591

Yeo G, Holste D, Kreiman G, Burge CB (2004) Variation in alternative splicing across human tissues. Genome Biol 5(10): R74

Yuan W, Zhou L, Chen JH, Wu JY, Rao Y, Ornitz DM (1999) The mouse SLIT family: secreted ligands for ROBO expressed in patterns that suggest a role in morphogenesis and axon guidance. Dev Biol 212(2):290-306 